\title{
CUMULANTS AND PARTITION LATTICES III: MULTIPLY-INDEXED ARRAYS
}

\author{
T. P. SPEED \\ (Received 13 January 1984) \\ Communicated by T. C. Brown
}

\begin{abstract}
Earlier work of the author exploiting the role of partition lattices and their Möbius functions in the theory of cumulants, $k$-statistics and their generalisations is extended to multiply-indexed arrays of random variables. The natural generalisations of cumulants and $k$-statistics to this context are shown to include components of variance and the associated linear combinations of mean-squares which are used to estimate them. Expressions for the generalised cumulants of arrays built up as sums of independent arrays of effects as in anova models are derived in terms of the generalized cumulants of the effects. The special case of degree two, covering the unbiased estimation of components of variance, is discussed in some detail.
\end{abstract}

1980 Mathematics subject classification (Amer. Math. Soc.): 62 A 05, $62 \mathrm{~J} 10$.

Keywords: cumulant, $k$-statistic, group action, anova model, component of variance.

\section{Introduction}

In two earlier papers with the same general title we have attempted to show that the recognition and exploitation of the partition lattices underlying the definitions of cumulants, $k$-statistics and their generalisations leads to more efficient and more general proofs of the main results. The aim of the present paper is to extend this work to multi-indexed arrays. We will find that most of the formulae of the earlier papers remain valid in the more general context, and the material we present forms the foundation for later papers in this series which deal with the asymptotics of variance component estimation and the computation of variances and covariances of variance component estimates.

(c) 1986 Australian Mathematical Society $0263-6115 / 86 \$ A 2.00+0.00$ 
The extension of Fisher's $k$-statistics and Tukey's polykays to samples from finite doubly-indexed populations was first given by Hooke $(1956 \mathrm{a}, \mathrm{b})$ in two papers which developed the theory of the relevant symmetric functions and applied it to the estimation of variance components. The generalised $k$-statistics Hooke defined were christened bipolykays. At around the same time there was a vigorous development of the theory of randomisation analyses of experimental designs using derived linear models and finite population framework. The initial work in this area is found in Kempthorne (1952), and it continued in a series of papers and Iowa theses by Wilk (1955), Wilk and Kempthorne (1956, 1957), Zyskind (1958, 1963), Throckmorton (1961), White (1963, 1975), Dayhoff (1964a, 1964b, 1966), Carney $(1967,1968,1970)$ and Kinney $(1971,1976)$. There were also close links between this theory and that of Cornfield and Tukey (1956) on anova models, and important unpublished work by Fairfield-Smith (1955).

As their primary interest was in the interpretation and validity of anova models and analyses, the early Iowa work concentrated upon first and second-degree expressions, whilst progressively generalising the underlying index set until the balanced complete populations or response structures were elucidated by Zyskind and Throckmorton. In this context certain key expressions emerged which were termed cap-sigmas by the Iowa school and canonical variance components by Fairfield-Smith; and Zyskind (1958) conjectured that these expressions should coincide with suitably generalised polykays of degree 2. Dayhoff (1964a, b) provided the generalisation and proved Zyskind's conjecture, whilst it was left to Carney (1967) to extend Hooke's work to arbitrary balanced complete response structures with a view to calculating the variances and covariances of estimated components of variance.

All of the work summarised so far took place in a finite population framework, with all random variables being obtained as suitably defined random samples. Furthermore, a number of the key results of Dayhoff (1964a, b, 1966) and Carney (1967, 1968) made use of an artifice known as "random cross labelling" (see White (1966, Section 5) for the detailed definition), whilst the practice well known to workers with cumulants of keeping all random variables distinct for maximum generality and simplicity was rarely adopted. As a consequence, whilst all the ingredients necessary for a complete and general presentation of the theory of generalised cumulants and $k$-statistics over arbitrary balanced complete response structures were present in the Iowa work, they have yet to be put together and studied in a manner wholly consistent with and generalising the single-index theory. This is the task of the present paper, and we turn now to a description of its contents.

We consider multiply-indexed arrays of indeterminates and random variables with an arbitrary system of nesting on the indices where we mean, loosely speaking, that an index $i_{q}$ is nested within an index $i_{p}$ (equivalently, $i_{p}$ nests $i_{q}$ ) if $i_{q}$ 
is only meaningful within the levels of $i_{p}$. The basic index set is $\mathbf{I}=\Pi\left\{\mathbf{I}_{p}: p \in P\right\}$ where $\mathbf{I}_{p}=\left\{1,2, \ldots, n_{p}\right\}, n_{p}$ finite or infinite, $p \in P$, with elements $i=\left(i_{p}: p \in\right.$ $P$ ), The relevant structure on the index set can be elucidated via a distributive lattice of commuting uniform equivalence relations (Speed and Bailey (1982)) or the generalised wreath product of the symmetric groups on the components $\mathbf{I}_{p}$, $p \in P$, relative to the partially ordered set $(P, \leqslant)$, where we write $q \leqslant p(p \geqslant q)$ if $i_{p}$ nests $i_{q}$; see Bailey et al. (1983) for this construction. When one or more of the sets $I_{p}$ is infinite, we restrict ourselves to the permutations of finite support, i.e. the finitary permutations, and form the restricted generalised wreath product, which simply means that whenever a set of maps into a group is defined by the construction, we consider only those maps which send all but finitely many points of their domain to the identity. It is through the action of this (restricted) generalised wreath product group $G=G W(\mathbf{I})$ of (restricted) symmetric groups that we recognise our context as coinciding with the Iowa school's balanced complete population structures.

Our first set of results in Section 2 give the definition of certain generalised symmetric functions, adapting the approach Doubilet (1972) used with the classical symmetric functions to our more general situation where the indeterminates are labelled by $\mathbf{I}$ and our symmetry is defined by $G=G W(\mathbf{I})$. In that section generalised $k$-statistics (more precisely, their coefficients) are defined, and the interrelations between them and the natural analogues of (augmented) monomial and power sum symmetric functions are derived. The formulae of Section 2 from paper II in this series continue to hold. This is also true for the basic facts concerning products of our generalised $k$-statistics, which are given in Section 3.

The next step is to give general definitions of generalised moments and cumulants which reduce to the appropriate finite and infinite population parameters. This is done in Section 4 below and the associated results concerning the unbiased estimates of these parameters are reviewed. All of this is exactly as with singly-indexed arrays, and the final two sections consider some results unique to our multi-indexed context, these being ones where the associated anova-type linear models play a role. In Section 5 we obtain expressions for the generalised cumulants of arrays which can be represented as linear models built up from independent components, whilst Section 6 discusses the second order theory and presents a more general version of Dayhoff's (1964b) equivalence theorem. We close with some remarks on the contents of later papers in this series.

\section{More generalised $k$-statistics}

We must preface our discussion of more generalised $k$-statistics with some results generalising the corresponding ones in Section 2 of (II). Any map $h$ : 
$\mathbf{m} \rightarrow \Pi\left\{\mathbf{n}_{p}: p \in P\right\}$ gives rise to further maps $h_{p}: \mathbf{m} \rightarrow \mathbf{n}_{p}, p \in P$, by composition with the coordinate projections, and we can thus define a map $p \rightarrow p^{h}(p)$ by the formula $\varphi^{h}(p)=\Lambda_{q \geqslant p} \operatorname{ker} h_{q}$. Clearly $\varphi^{h} \in \operatorname{Hom}(P, \mathscr{P}(\mathbf{m}))$, the lattice of all order-preserving maps from $P$ into $\mathscr{P}(\mathbf{m})$. Now let us consider $\pi \in \operatorname{Hom}(P, \mathscr{P}(\mathbf{m}))$ and write

$$
\mathcal{O}_{n}=\left\{h \in \mathbf{I}^{\mathbf{m}}: \varphi^{h}=\pi\right\} .
$$

The following lemma is proved in Praeger et al. (1985).

LEMMA 2.1. (i) $\left\{\mathcal{O}_{\pi}: \pi \in \operatorname{Hom}(P, \mathscr{P}(\mathbf{m}))\right\}$ is a partition of $\Pi\left\{\mathbf{n}_{p}: p \in P\right\}^{\mathrm{m}}$.

(ii) $\mathcal{O}_{\pi}$ is $G W(\mathbf{I})$-invariant, $\pi \in \operatorname{Hom}(P, \mathscr{P}(\mathbf{m}))$.

(iii) $G W(\mathbf{I})$ acts transitively on $\mathcal{O}_{\pi}, \pi \in \operatorname{Hom}(P, \mathscr{P}(\mathbf{m}))$.

It follows from this lemma that our symmetric functions will be labelled by elements of the lattice $\operatorname{Hom}(P, \mathscr{P}(\mathbf{m}))$ for a given order $m$. This result is essentially in Carney (1968, Section 7) where the notion of admissible $|P|$-fold ordered partition of weight $m$ introduced there in Definition 7.2 corresponds to an element of $\operatorname{Hom}(P, \mathscr{P}(\mathrm{m}))$. We now need to state certain results concerning the Möbius function of this lattice and the analogues of the decreasing powers $(n)_{\sigma}, \sigma \in \mathscr{P}(\mathrm{m})$, which played such a crucial role in our earlier discussion of generalised $k$-statistics. As before these numbers give the cardinalities of the orbits, i.e. $\left|\mathcal{O}_{\sigma}\right|=(n)_{\sigma}$.

The following lemma is proved in Speed (1984) and may be used to obtain the Möbius function of $\operatorname{Hom}(P, \mathscr{P}(\mathbf{m}))$.

LEMMA 2.2. Let $P$ and $Q$ be arbitrary posets with Möbius and zeta functions $\mu_{P}$, $\mu_{Q}, \zeta_{P}$ and $\zeta_{Q}$ respectively. Then the Möbius function $\mu$ of $\operatorname{Hom}(P, Q)$ is given by

$$
\mu(\varphi, \psi)=\prod_{p \in P}\left\{\mu_{Q}(\varphi(p), \psi(p)) \prod_{p^{\prime}>p} \zeta_{Q}\left(\psi(p), \varphi\left(p^{\prime}\right)\right)\right\} .
$$

The roles of the normal and descending powers $n^{\sigma}$ and $(n)_{\sigma}$ corresponding to $\sigma \in \mathscr{P}(\mathbf{m})$ are taken here by the function $n^{\sigma}$ and $(n)_{\sigma}, \sigma \in \operatorname{Hom}(P, \mathscr{P}(\mathbf{m}))$, where

$$
n^{\sigma}:=\prod_{p \in P} n_{p}^{\sigma(p)}=\prod_{p \in P} n_{p}^{b(\sigma(p))},
$$

and

$$
(n)_{\sigma}=\prod_{p \in P}\left(n_{p}\right)_{(\sigma(p), \wedge\{\sigma(q): q>p\})}
$$

and we are using the notation $(k)_{(\rho, \tau)}=\sum_{\sigma} \mu(\rho, \sigma) \zeta(\sigma, \tau) k^{\sigma}, \rho, \tau \in \mathscr{P}(\mathbf{m}), k \in \mathbb{N}$. The following result is essentially in Carney (1967, V B Section 2), although a proof in our current framework can be found in Speed (1984). 
LEMMA 2.3. In the lattice $\operatorname{Hom}(P, \mathscr{P}(\mathrm{m}))$ we have

$$
n^{\varphi}=\sum_{\psi} \zeta(\varphi, \psi)(n)_{\psi} ; \quad(n)_{\varphi}=\sum_{\psi} \mu(\varphi, \psi) n^{\psi}
$$

Now that we have organised the combinatorial preliminaries, it is a quite straightforward task to generalise the notions introduced in (II) to the present situation.

The coefficient tensors which are our more generalised $k$-statistics (which include poly-bi-kays and bi-polykays etc.) are defined using the basis $\left\{\delta^{i}: i \in \mathbf{I}\right\}$ where $\delta^{i}=\otimes_{p \in P} \delta^{i_{p}}, i \in \mathbf{I}, \boldsymbol{\delta}^{i_{p}}$ denoting the $n_{i_{p}} \times 1$ basis vector of the kind introduced in (II). For $h: \mathbf{m} \rightarrow \mathbf{I}$ with $\varphi^{h} \in \operatorname{Hom}(P, \mathscr{P}(\mathbf{m}))$ we define $\bar{h}$ just as before, viz

$$
\bar{h}=\delta^{h(1)} \otimes \cdots \otimes \delta^{h(m)}
$$

and, again as before, we define $A_{n}, R_{\pi}$ and $F_{\pi}$ for $\pi \in \operatorname{Hom}(P, \mathscr{P}(\mathrm{m}))$ by $A_{\pi}=\sum_{\phi^{h}=\pi} \bar{h}$, abbreviated $(n)_{\pi}\langle\pi\rangle ;$ and $R_{\pi}=\sum_{\varphi^{h} \geqslant \pi} \bar{h}$, abbreviated $[\pi]$; the generalised $k$-statistic in this context being $F_{\pi}=\Sigma_{\sigma} \mu(\sigma, \pi)(n)_{\sigma}^{-1} A_{\sigma}$, abbreviated $(\pi)$.

Proposition 2.1. The transition matrices $M(A, R), M(F, A), M(F, R)$ and their inverses are given by exactly the same formulae as in Proposition 2.1 of (II) interpreted in $\operatorname{Hom}(P, \mathscr{P}(\mathbf{m}))$.

Now let us suppose that $x=\left(x_{i}(l): l \in \mathbf{m}, i \in \mathbf{I}\right)$ is an array of indeterminates. As before we can view $x$ as an array $\left(x_{i}: i \in I\right)$ of $m$-tuple indeterminates labelled by $\mathbf{I}$, or as an array $(x(1), \ldots, x(m))$ of $\Pi_{p \in P} n_{p}$-tuple indeterminates (indexed lexicographically) labelled by $\mathbf{m}$. With this second view in mind we define $x=x(1) \otimes \cdots \otimes x(m)$ and the associated generalised symmetric functions $a_{\pi}=$ $\left[A_{\pi} \mid x\right], s_{\pi}=\left[R_{\pi} \mid x\right]$, and $k_{\pi}=\left[F_{\pi} \mid x\right]$. By their construction each of these polynomials is seen to be invariant under $G W(\mathbf{I})$. At times we will write $k(\pi), a(\pi)$, $k_{n_{1}, \ldots, n_{s}}(\pi)$ etc. where $s=|P|$.

$A$ remark on the relation between our notation and that of earlier workers seems to be in order although we refer to the papers concerned for general definition of their notation. For the singly nested structure $P=1 \frac{1}{2}$ and $m=2$ we have three sets of coefficients labelled by pairs $\pi(1), \pi(2)$ from $\mathscr{P}(2)$ with $\pi(1) \geqslant \pi(2)$, and some of these are tabulated below with their analogues from Carney (1968). It would take too much space to give the corresponding notations from Dayhoff $(1964 a, b)$ and Hooke $(1956 a, b)$ but these are not difficult to relate to those given below. In Table $1, \pi(1), \pi(2)$ may be 12 or $1 / 2$ whilst $\alpha^{1}, \alpha^{2}$ may be 11 or 10 , subject to the constraints arising from the nesting. 


\section{Present paper*}

(i) augmented monomial symmetric:

coefficients: $A_{\pi(1), \pi(2)}$

function: $a_{\pi(1), \pi(2)}$

(ii) scaled version of (i):

coefficients:

$\langle\pi(1), \pi(2)\rangle=\frac{1}{\left(n_{1}\right)_{\pi(1)}\left(n_{2}\right)_{(\pi(2), \pi(1))}} A_{\pi(1), \pi(2)}$

function: $k_{\pi(1), \pi(2)}$

$$
\frac{1}{\left(n_{1}\right)_{\pi(1)}\left(n_{2}\right)_{(\pi(2), \pi(1))}} a_{\pi(1), \pi(2)}
$$

(iii) power sum symmetric:

coefficients: $[\pi(1), \pi(2)]=R_{\pi(1), \pi(2)}$

function: $s_{\pi(1), \pi(2)}$

(iv) $k$-statistics:

polykay: $\left(\boldsymbol{\alpha}^{1} / \boldsymbol{\alpha}^{2}\right)$

coefficients: $(\pi(1), \pi(2))=F_{\pi(1), \pi(2)}$

Carney $^{\dagger}$

symmetric sum: $\left|\alpha^{1} / \alpha^{2}\right|$

symmetric mean: $\left\langle\alpha^{1} / \alpha^{2}\right\rangle$

function: $k_{\pi(1), \pi(2)}$

${ }^{*}$ Here $\pi(1)$ and $\pi(2)$ are partitions of $2=\{1,2\}$ with $\pi(1) \geqslant \pi(2)$. $\nmid \alpha^{1}$ and $\alpha^{2}$ are ordered partitions of weight 2 (see Carney (1968) for details) subject to $\alpha^{1} \geqslant \alpha^{2}$.

TABLE 1

Example 2.1. $P=\left\lfloor\frac{1}{2}, m=2\right.$. The lattice $\operatorname{Hom}(P, 2)$ has three elements $1 \rightarrow 12$, $2 \rightarrow 12 ; 1 \rightarrow 12,2 \rightarrow 1 \mid 2$ and $1 \rightarrow 1|2,2 \rightarrow 2| 2$ which we write $(12,12),(12,1 \mid 2)$ and $(1|2,1| 2)$ respectively; see Figure 1 .

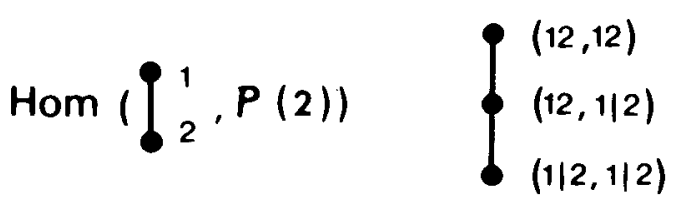

Figure 1

These correspond to the pairs $(i j, i j),\left(i j, i j^{\prime}\right)$ with $j^{\prime} \neq j$, and $\left(i j, i^{\prime} j^{\prime}\right)$ with $i^{\prime} \neq i$ (but not necessarily $j^{\prime} \neq j$ ). We will use $\left(x_{i j}, y_{i j}\right)$ in this case instead of $\left(x_{i_{1} i_{2}}(1), x_{i_{1} i_{2}}(2)\right)$.

With this notation we readily find that

$$
\begin{aligned}
& \langle 12,12\rangle=\frac{1}{n_{1} n_{2}} \sum \delta^{i j} \otimes \delta^{i j}, \\
& \langle 12,1 \mid 2\rangle=\frac{1}{n_{1} n_{2}\left(n_{2}-1\right)} \sum \delta^{i j} \otimes \delta^{i j^{\prime}}
\end{aligned}
$$


summed over all $i, j$ and $j^{\prime} \neq j$, whilst

$$
\langle 1|2,1| 2\rangle=\frac{1}{n_{1}\left(n_{1}-1\right) n_{2}^{2}} \sum \delta^{i j} \otimes \delta^{i^{\prime} j^{\prime}}
$$

is summed over all $i, i^{\prime} \neq i, j$ and $j^{\prime}$. The corresponding expressions $[12,12]$, $[12,1 \mid 2]$ and $[1|2,1| 2]$ are simply obtained by omitting the inequality constraints on the indices. Thus we can use the Möbius function of the lattice in Figure 1 to derive

$$
\begin{aligned}
(12,12) & =\langle 12,12\rangle-\langle 12,1 \mid 2\rangle \\
& =\frac{1}{n_{1} n_{2}}[12,12]+\frac{1}{n_{1} n_{2}\left(n_{2}-1\right)}\{[12,1 \mid 2]-[12,12]\} \\
& =\frac{1}{n_{1}\left(n_{2}-1\right)}\left\{[12,12]-\frac{1}{n_{2}}[12,1 \mid 2]\right\} .
\end{aligned}
$$

Substituting an array $\left\{\left(x_{i j}, y_{i j}\right):(i, j) \in \mathbf{n}_{1} \times \mathbf{n}_{2}\right\}$ of indeterminates and simplifying, we obtain

$$
\begin{aligned}
k(12,12) & =[(12,12) \mid x \otimes y] \\
& =\frac{1}{n_{1}\left(n_{2}-1\right)}\left\{[[12,12] \mid x \otimes y]-\frac{1}{n_{2}}[[12,1 \mid 2] \mid x \otimes y]\right\} \\
& =\frac{1}{n_{1}\left(n_{2}-1\right)}\left\{\sum_{i} \sum_{j} x_{i j} y_{i j}-\frac{1}{n_{2}} \sum_{i} \sum_{j} \sum_{j^{\prime}} x_{i j} y_{i j^{\prime}}\right\} \\
& =\frac{1}{n_{1}\left(n_{2}-1\right)} \sum_{i} \sum_{j}\left(x_{i j}-x_{i .}\right)\left(y_{i j}-y_{i .}\right)
\end{aligned}
$$

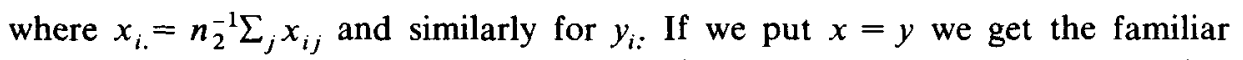
within groups mean square in a singly-nested anova decomposition, a connection which will be fully explained later.

Similarly it would be found that

$$
\begin{aligned}
(12,1 \mid 2)= & \langle 12,1 \mid 2\rangle-\langle 1|2,1| 2\rangle \\
= & \frac{1}{n_{1} n_{2}\left(n_{2}-1\right)}\{[12,1 \mid 2]-[12,12]\} \\
& -\frac{1}{n_{1}\left(n_{1}-1\right) n_{2}^{2}}\{[1|2,1| 2]-[12,1 \mid 2]\} \\
= & \frac{-1}{n_{1} n_{2}\left(n_{2}-1\right)}[12,12]+\frac{1}{n_{1} n_{2}}\left\{\frac{1}{n_{2}-1}+\frac{1}{n_{2}\left(n_{1}-1\right)}\right\}[12,1 \mid 2] \\
& -\frac{1}{n_{1}\left(n_{2}-1\right) n_{2}^{2}}[1|2,1| 2]
\end{aligned}
$$


whence

$$
\begin{aligned}
k(12,1 \mid 2)= & \frac{-1}{n_{1} n_{2}\left(n_{2}-1\right)} \sum_{i} \sum_{j} x_{i j} y_{i j} \\
& +\frac{1}{n_{1} n_{2}}\left\{\frac{1}{n_{2}-1}+\frac{1}{n_{2}\left(n_{1}-1\right)}\right\} \sum_{i} \sum_{j} \sum_{j^{\prime}} x_{i j} x_{i j^{\prime}} \\
& -\frac{1}{n_{1}\left(n_{1}-1\right) n_{2}^{2}} \sum_{i} \sum_{i^{\prime}} \sum_{j} \sum_{j^{\prime}} x_{i j} x_{i^{\prime} j^{\prime}}
\end{aligned}
$$

Although it is not obvious, and there is no general theory covering such simplifications, it is not hard to show that this does simplify to

$$
\begin{aligned}
k(12,1 \mid 2)=\frac{1}{n_{2}} & \left\{\frac{n_{2}}{n_{1}-1} \sum_{i}\left(x_{i .}-x . .\right)\left(y_{i .}-y_{. .}\right)\right. \\
& \left.\quad-\frac{1}{n_{1}\left(n_{2}-1\right)} \sum_{i} \sum_{j}\left(x_{i j}-x_{i .}\right)\left(y_{i j}-y_{i .}\right)\right\}
\end{aligned}
$$

When $x=y$ this is seen to be the usual linear combination of the between and within mean squares from the anova which unbiasedly estimates the between groups variance component under the usual additive linear model.

Now let us consider $m=3$. In this case the lattice $\operatorname{Hom}(P, \mathscr{P}(3))$ has 12 elements $(\pi(1), \pi(2))$ with $\pi(1), \pi(2) \in \mathscr{P}(3)$ satisfying $\pi(1) \geqslant \pi(2)$; see Figure 2 .

Referring to Figure 2 to calculate the values of the Möbius function, we expand $(123,123)$ as follows:

$$
\begin{aligned}
(123,123)= & \langle 123,123\rangle-\langle 123,1 \mid 23\rangle-\langle 123,2 \mid 13\rangle \\
& -\langle 123,3 \mid 12\rangle+2\langle 123,1|2| 3 \mid\rangle \\
= & \frac{1}{n_{1} n_{2}}[123,123]-\frac{1}{n_{1} n_{2}\left(n_{2}-1\right)}\{[123,1 \mid 23]-[123,123]\} \\
& -2 \text { similar terms } \\
& +\frac{1}{n_{1} n_{2}\left(n_{2}-1\right)\left(n_{2}-2\right)}\{[123,1|2| 3]-[123,1 \mid 23]-[123,2 \mid 13] \\
= & \frac{n_{2}}{n_{1}\left(n_{2}-1\right)\left(n_{2}-2\right)}\left\{[123,123]-\frac{1}{n_{2}}[123,1 \mid 23]-\frac{1}{n_{2}}[123,2 \mid 13]\right. \\
& \left.-\frac{1}{n_{2}}[123,3 \mid 12]+\frac{2}{n_{2}^{2}}[123,1 \mid 2,3]\right\} .
\end{aligned}
$$




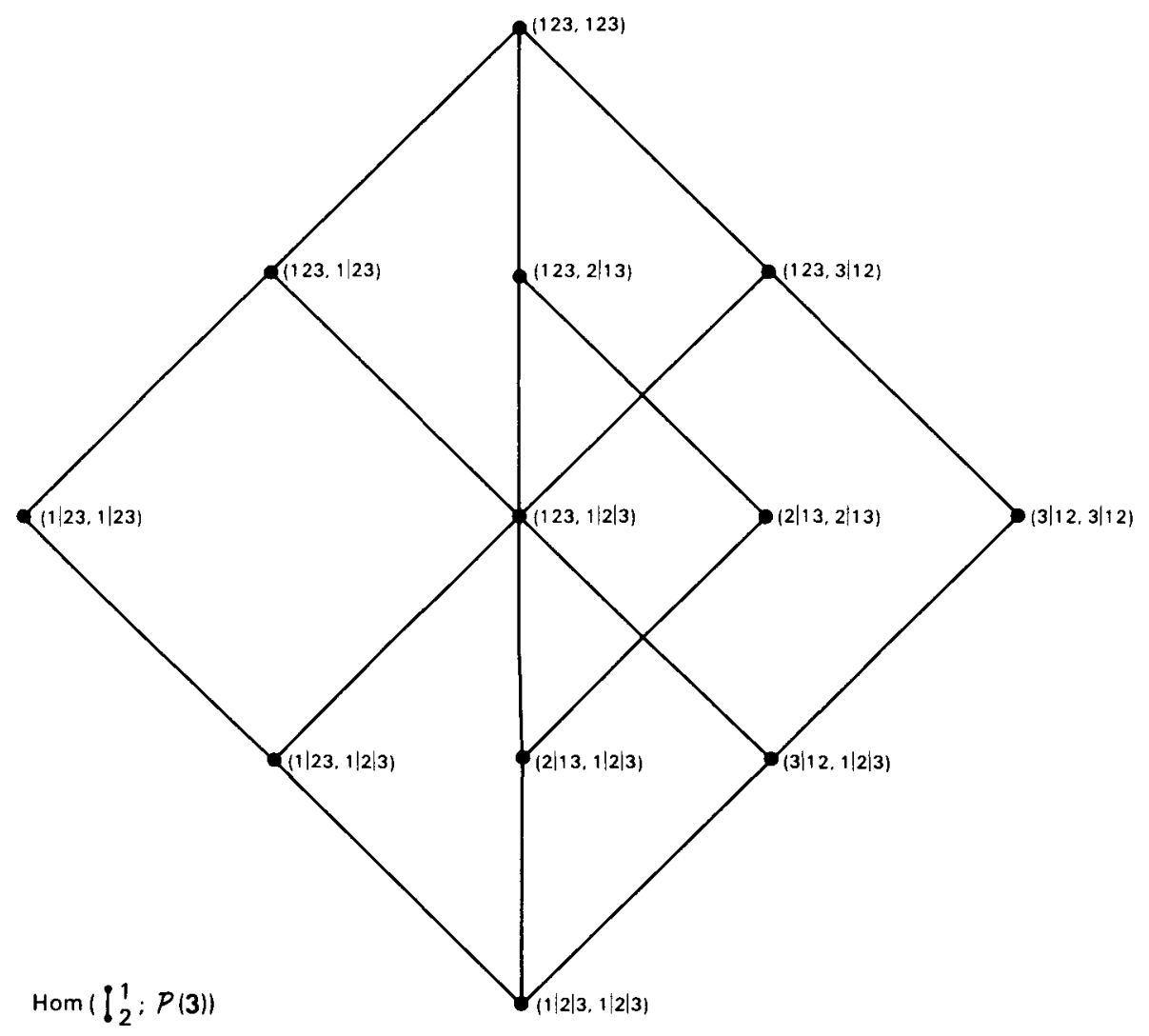

Figure 2

Evaluating this for an array $\left(\left(x_{i j}, y_{i j}, z_{i j}\right):(i, j) \in \mathbf{n}_{1} \times \mathbf{n}_{2}\right)$ and simplifying we find that the symmetric function (which, following Tukey we might term the within class (cross) skewmulance) has the form

$$
k(123,123)=\frac{n_{2}}{n_{1}\left(n_{2}-1\right)\left(n_{2}-2\right)}\left\{\sum_{i=1}^{n_{1}} \sum_{j=1}^{n_{2}}\left(x_{i j}-x_{i .}\right)\left(y_{i j}-y_{i .}\right)\left(z_{i j}-z_{i .}\right)\right\} \text {. }
$$

Once more we remark that no general theory of such simplications exists although the expression clearly has an intuitively reasonable form and will relate in a natural way to a linear model for the array of which the details will be explained in Section 5 below.

\section{Products of more generalised $k$-statistics}

One of the great benefits of formulating our results in terms of the lattices $\mathscr{P}(\mathbf{m})$ and $\operatorname{Hom}(P, \mathscr{P}(\mathbf{m}))$ is the fact that most proofs generalise immediately. 
Just as with Proposition 2.1 above we have an analogue of Proposition 3.1 of (II). The notation is that of Section 3 of (II) and we simply define restrictions coordinatewise, e.g. for $\rho \in \operatorname{Hom}(P, \mathscr{P}(\mathbf{m}))$ we define $\rho \cap \mathbf{m}_{i}$ to be $p \rightarrow \rho(p) \cap$ $\mathbf{m}_{i}, p \in P$.

Proposition 3.1. For $\sigma_{1} \in \operatorname{Hom}\left(P, \mathscr{P}\left(\mathbf{m}_{1}\right)\right), \ldots, \sigma_{r} \in \operatorname{Hom}\left(P, \mathscr{P}\left(\mathbf{m}_{r}\right)\right)$ we have

$$
\left(\sigma_{1}\right) \otimes \cdots \otimes\left(\sigma_{r}\right)=\sum_{\sigma} c\left(\sigma ; \sigma_{1}, \ldots, \sigma_{r}\right)(\sigma)
$$

where the sum is over all $\sigma \in \operatorname{Hom}(P, \mathscr{P}(\mathbf{m}))$ and

$$
c\left(\sigma ; \sigma_{1}, \ldots, \sigma_{r}\right)=\sum_{\rho \geqslant \sigma}(n)_{\rho} \prod_{i=1}^{r} \frac{\mu\left(\rho \cap \mathbf{m}_{i}, \boldsymbol{\sigma}_{i}\right)}{(n)_{\rho \cap \mathbf{m}_{i}}} .
$$

Proof. This can be proved exactly as Proposition 3.1 of (II).

Another advantage of working with the full lattice $\operatorname{Hom}(P, \mathscr{P}(\mathbf{m}))$ rather than the sub-structure which arises when one or more of the $m$ indeterminates is identified is that we get the following proposition. It was almost but not quite evident in the work of Hooke $(1956 \mathrm{a}, \mathrm{b})$ who studied the case $P=i$, but only ever used a single array of indeterminates.

Proposition 3.2. (Crossing Rule). If $P$ is a set of pairwise incomparable elements, then for any $\sigma_{1} \in \operatorname{Hom}\left(P, \mathscr{P}\left(\mathbf{m}_{1}\right)\right), \ldots, \sigma_{r} \in \operatorname{Hom}\left(P, \mathscr{P}\left(\mathbf{m}_{r}\right)\right)$, and $\boldsymbol{\sigma} \in$ $\operatorname{Hom}(P, \mathscr{P}(\mathbf{m}))$ we have

$$
c\left(\sigma ; \sigma_{1}, \ldots, \sigma_{r}\right)=\prod_{p \in P} c\left(\sigma(p) ; \sigma_{1}(p), \ldots, \sigma_{r}(p)\right) .
$$

Proof. Under the hypotheses of the proposition, $\operatorname{Hom}(P, \mathscr{P}(\mathbf{m})) \cong \mathscr{P}(\mathbf{m})^{|P|}$ and its Möbius function is just a product of Möbius functions of $\mathscr{P}(\mathbf{m})$. Thus $(n)_{\sigma}=\prod_{p \in P}\left(n_{p}\right)_{\sigma(p)}$ and the result follows.

EXAMPLE 3.1. Suppose that we wish to compute $(12,12) \otimes(34,3 \mid 4)$ in $\operatorname{Hom}\left({ }_{i 2}, \mathscr{P}(4)\right)$. By the preceding proposition the following formalism is justified; see Section 3 of (II) for the single-index results used.

$$
\begin{aligned}
(12,12) & \otimes(34,3 \mid 4)=((12) \otimes(34),(12) \otimes(3 \mid 4)) \\
= & \left(\frac{1}{n_{1}}(1234)+(12 \mid 34)+\frac{1}{n_{1}-1}\{(13 \mid 24)+(14 \mid 23)\},\right. \\
\left.\frac{1}{n_{2}}\{(123 \mid 4)+(124 \mid 3)\}+\cdots\right) & \\
= & \frac{1}{n_{1} n_{2}}(1234,123 \mid 4)+\frac{1}{n_{1} n_{2}}(1234,124 \mid 3)+\frac{1}{n_{2}}(12|34,123| 4)+\text { etc. }
\end{aligned}
$$


It would be very good indeed if there was a product formula generalising Proposition 3.2 to arbitrary posets $P$ which expresses the coefficient $c\left(\sigma ; \sigma_{1}, \ldots, \sigma_{r}\right)$ in some way as a product of "one-factor" $c$-coefficients, but one of the big disappointments of this work was the realisation that no such formula exists. We illustrate this with the simplest non-crossed structure.

Example 3.2. The coefficient $c((13|24,13| 24) ;(12,1 \mid 2),(34,3 \mid 4))$ of $(13|24,13| 24)$ in the expansion of $(12,1 \mid 2) \otimes(34,3 \mid 4)$ turns out to be

$$
\frac{1}{n_{1} n_{2}\left(n_{2}-1\right)}+\frac{1}{n_{1} n_{2}^{2}\left(n_{1}-1\right)}=\frac{n_{1} n_{2}-1}{n_{1} n_{2}^{2}\left(n_{1}-1\right)\left(n_{2}-1\right)}
$$

which is clearly not of product form.

The only thing that remains is the tabulation of product expansions. At the moment we have only done this for the products $\pi \otimes \sigma$ where $\pi, \sigma \in$ $\operatorname{Hom}(P, \mathscr{P}(2))$ and where $P$ is

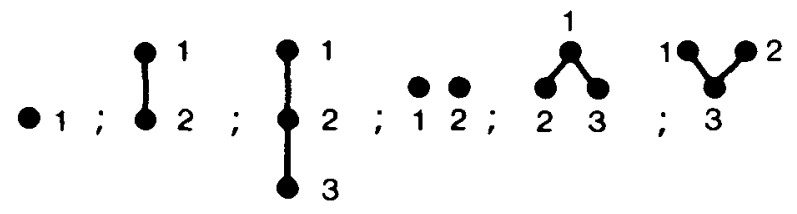

The results will be reported in a later paper in this series in which the variances and covariances of the usual unbiased estimates of components of variance are discussed. See also Section 6 below.

\section{More generalised moments and cumulants}

We now extend the definitions and results of Section 4 of (II) to apply to random arrays $X=\left(X_{i}: i \in \mathbf{I}\right)=(X(l): l \in \mathbf{m})$ whose moments of order $m$ are invariant under the action of $G=G W(\mathbf{I})$ on $\mathbf{I}=\Pi\left\{\mathbf{n}_{p}: p \in P\right\}$. Note that Definition 4.1 of (II) applies here without change. A good deal of the material in this section is a direct extension of corresponding material in (II) and so we pass over that very quickly, dwelling only on those aspects which are specific to the more general framework: linear models, linear decompositions and the theory of variance components for complete balanced response structures.

Just as we saw in (II) there are two main ways in which random arrays $X$ with $G W$-invariant moments arise: through independence, and through symmetric random sampling. The analogues here of the iid arrays of (II) are those arrays which are built up as sums of mutually independent arrays or, somewhat more precisely, as sums from sets of arrays whose elements are mutually independent between and have symmetric moments within sets. We discuss such arrays in 
detail below. The notion of the $m$-symmetric random sampling distribution associated with an array $\tilde{X}=\left(\tilde{X}_{I}: I \in \tilde{\mathbf{I}}\right)$ of real numbers is defined here in essentially the same way as in (II) Section 4 where we had $\mathbf{I}=\mathbf{n}$ and $\tilde{\mathbf{I}}=\mathbf{N}$, $n \leqslant N$. In the present framework we suppose that the population array $\tilde{X}$ is indexed by $\tilde{\mathbf{I}}=\Pi\left\{\mathbf{N}_{p}: p \in P\right\}$, where $n_{p} \leqslant N_{p}$ for each $p \in P$. Now every $m$ tuple $\left(i_{1}, \ldots, i_{m}\right) \in \mathbf{I}^{\mathbf{m}}$ belongs to a unique orbit, $\mathcal{O}_{\pi}$ say, $\pi \in \operatorname{Hom}(P, \mathscr{P}(\mathbf{m}))$ of $G W(\mathbf{I})$ acting on $\mathbf{I}^{\mathbf{m}}$, and we denote $\pi$ by $\pi\left[i_{1}, \ldots, i_{m}\right]$ (not to be confused with $\pi(p)$, the image of $p \in P$ under $\pi)$. Similarly we can define $\pi\left[I_{1}, \ldots, I_{m}\right]$ for every $m$-tuple $\left(I_{1}, \ldots, I_{m}\right) \in \tilde{\mathbf{I}}^{m}$, and with these preliminaries we say that the array $X$ has $m$-invariant inclusion probabilities (under $G W$ ) if for every $m$-tuple $\left(i_{1}, \ldots, i_{m}\right) \in \mathbf{I}^{\mathbf{m}}$ we have

$$
\operatorname{pr}\left(X_{i_{1}}=\tilde{X}_{I_{1}}, \ldots, X_{i_{m}}=\tilde{X}_{I_{m}}\right)= \begin{cases}\frac{1}{(N)_{\pi}} & \text { if } \pi\left[i_{1}, \ldots, i_{m}\right]=\pi\left[I_{1}, \ldots, I_{m}\right]=\pi, \\ 0 & \text { otherwise. }\end{cases}
$$

In other words, we ask for uniform distribution over the orbits of $G W(\tilde{\mathbf{I}})$. Although it was not formalised to this extent, it is essentially this type of probability model that underlies all of the work of the Iowa school beginning with Kempthorne (1952), through to Carney (1967) and beyond, i.e. their randomisation analyses view the observed array $X$ as a sample from a finite population $\tilde{X}$; similarly with Hooke (1956a, b), Cornfield and Tukey (1956) and others.

EXAmple 4.1. Suppose that $P=?$ ? and that we let $m=2$, writing $\left(\left(X_{i j}, Y_{i j}\right):(i, j) \in \mathbf{r} \times \mathbf{c}\right)$ for the sampled array and $\left(\left(\tilde{X}_{I J}, \tilde{Y}_{I J}\right):(I, J) \in \mathbf{R} \times \mathbf{C}\right)$ for the population array. The independent sampling of $r$ rows from the $\mathbf{R}$ and $c$ columns from the $\mathrm{C}$ in the population, and then using the $r c$ cells so defined clearly satisfies (4.1). As an illustration, let us note that such a procedure would clearly imply that when $j \neq j^{\prime}$ :

$$
\operatorname{pr}\left(X_{i j}=\tilde{X}_{I J}, X_{i j^{\prime}}=\tilde{X}_{I^{\prime} J^{\prime}}\right)= \begin{cases}\frac{1}{R C(C-1)} & \text { if } I=I^{\prime}, \quad J \neq J^{\prime} \\ 0 & \text { otherwise }\end{cases}
$$

Furthermore $\pi\left(i j, i j^{\prime}\right)=(12,1 \mid 2)$ and for this $\pi,(N)_{\pi}=R C(C-1)$.

The definitions of the generalised moments $\gamma_{\sigma}$ and generalised cumulant $f_{\sigma}$ of order $m$ of an array $X$ with $G W$-invariant moments are exact analogues of those given in Definition 4.2 of (II) except that here $\sigma \in \operatorname{Hom}(P, \mathscr{P}(\mathbf{m}))$ and we make use of a map $h: \mathbf{m} \rightarrow \mathbf{I}=\prod\left\{\mathbf{n}_{p}: p \in P\right\}$ with $\varphi^{h}=\boldsymbol{\sigma}$. We will not repeat the details. The specialised forms $\gamma_{\sigma}$ and $f_{\sigma}$ take when $X$ is defined by a linear model 
with independent components will be derived below. For completeness we state the following analogue of Proposition 4.1 of (II).

Proposition 4.1. If $X$ is a random array with $G W(\mathbf{I})$-invariant moments of order $m$, then

$$
\mathbb{E}\{X\}=\sum_{\sigma} \gamma_{\sigma} A_{\sigma}=\sum_{\rho} f_{\rho} R_{\rho}
$$

where the sums are over $\operatorname{Hom}(P, \mathscr{P}(\mathbf{m}))$.

How are we to interpret these (more) generalised moments and cumulants? For $|P|=1$ we have already partially answered this question, but what about arbitrary $P$ ? We will see below that for $P$ arbitrary and $m=2$, the generalised cumulants are in fact the components of variance known as canonical (see Fairfield-Smith (1955)) or cap-sigmas, the latter term being used by the Iowa school. When the array $X$ is built up from independent arrays of effects in linear models, these generalised cumulants will turn out to be sums of products of cumulants of the component effects. It is not clear how useful the generalised cumulants are beyond $m=4$; even for $m \leqslant 4$ their expressions can be quite complex.

Without further ado we state the generalised forms of Propositions 4.2 and 4.3 of (II) in a single result. The proof is identical to that of the earlier ones, whilst the notation is that of Section 3 above.

Proposition 4.2 Let $X$ be a random array with $G W$-invariant moments of order $m$. Then for all $\mathrm{\sigma} \in \operatorname{Hom}(P, \mathscr{P}(\mathbf{m}))$ we have

$$
\mathbb{E}\left\{(n)_{\sigma}^{-1} a_{\sigma}\right\}=\gamma_{\sigma}, \quad \mathbb{E}\left\{k_{\sigma}\right\}=f_{\sigma} .
$$

If $X$ has $G W$-invariant moments of order $2 m$, then $(n)_{\sigma}^{-1} a_{\sigma}$ and $k_{\sigma}$ have minimum variance amongst all polynomials of degree $m$ in $X_{i}(l): l \in \mathbf{m}, i \in \mathbf{I}$ which are unbiased estimators of $\gamma_{\sigma}$ and $f_{\sigma}$ respectively.

\section{Linear models for random arrays}

It is known from the theory associated with the analysis of variance (see e.g. Speed (1986)) that any random array $X=\left(X_{i}: i \in \mathbf{I}\right)$ with $G W(\mathbf{I})$-invariant moments of order 2 can be decomposed as follows:

$$
X_{i}=\sum_{a \in F(P)} \varepsilon_{i}(a)
$$

where

(i) $F(P)$ is the distributive lattice of all filters (= dual ideals) of $P$, which is anti-isomorphic with $\operatorname{Hom}(P, \mathscr{P}(2))$; 
(ii) elements of the random array $\left(\varepsilon_{i}(a): i \in I\right)$ depend on $i$ only through $i_{p}$, $p \in a$, i.e. $\varepsilon_{i}(a)=\varepsilon_{j}(a)$ if $i_{p}=j_{p}, p \in a$; and

(iii) for each pair $i, j \in \mathbf{I}$ and $a \neq b \in F(P), \varepsilon_{i}(a)$ is uncorrelated with $\varepsilon_{j}(b)$. Furthermore, if $n_{p} \equiv \infty$, then we also have the conclusion:

(iv) for each $a \in F(P)$ and pair $i, j \in \mathbf{I}$ which differ over $a, \varepsilon_{i}(a)$ and $\varepsilon_{j}(a)$ are uncorrelated and have equal variance.

Equation (5.1) is the spectral or principal components decomposition of $X$ resulting from the form of its covariance matrix. Bearing in mind the fact that zero correlation implies independence under normality, it is not surprising that many writers have built up random arrays $X$ in the manner described in (i) to (iv) from component effects $\left(\varepsilon_{i}(a)\right)$, but replacing zero correlation by independence. We will now derive expressions for the generalised moments and cumulants of such synthesized arrays $X$ in terms of those of the component arrays, $a \in F(P)$. It will be convenient to suppose that the single random variable $\varepsilon_{i}(\varnothing)$ is constant, $\mu$ say, and that all the other $\varepsilon$ 's have mean zero. Our main result will follow a sequence of three lemmas, each generalising a similar result in Hooke (1956a) where the case $P=1$ ? was studied.

The first lemma gives the generalised moments and cumulants $\gamma^{X}(\tau)$ and $f^{X}(\tau)$ of $X$ in terms of those of similarly indexed arrays $Y$ and $Z$, where we suppose that the arrays $Y$ and $Z$ are independent and that $X_{i}=Y_{i}+Z_{i}, i \in \mathbf{I}$. It generalises the corollary to Proposition 4.2 of (I) and Theorem 4 of Hooke (1956a). Here $\tau=(\tau(p): p \in P) \in \operatorname{Hom}(P, \mathscr{P}(\mathbf{m}))$.

LEMMA 5.1. (i) $\gamma^{X}(\sigma)=\sum_{s \subseteq m} \gamma^{Y}(\sigma \cap s) \gamma^{Z}\left(\sigma \cap s^{\prime}\right), s^{\prime}=\mathbf{m} \backslash s$

(ii) $f^{X}(\tau)=\sum_{\rho \mid \sigma=\tau} f^{Y}(\rho) f^{Z}(\sigma)$,

the second sum being over all pairs $\rho, \sigma \in \operatorname{Hom}(P, \mathscr{P}(\mathbf{m}))$ for which $\rho \mid \sigma=\tau$ (componentwise), and including the empty partition for which the f's take the conventional value 1 .

Proof. For any $h: \mathbf{m} \rightarrow \mathbf{I}$ with $\varphi^{h}=\sigma$ we have

$$
\begin{aligned}
\prod_{l \in \mathbf{m}} X_{h(l)}(l) & =\prod_{l \in \mathbf{m}}\left\{Y_{h(l)}(l)+Z_{h(l)}(l)\right\} \\
& =\sum_{s \subseteq \mathbf{m}} \prod_{k \in s} Y_{h(k)}(k) \prod_{l \in s^{\prime}} Z_{h(l)}(l)
\end{aligned}
$$

and so (i) is an immediate consequence of the independence of $Y$ and $Z$.

To prove (ii) we make use of (i) and write $\gamma^{Y}(\sigma \cap s)$ as follows:

$$
\gamma^{Y}(\sigma \cap s)=\sum_{\pi} \zeta(\pi, \sigma \cap s) f^{Y}(\pi)
$$


the sum being over all $\pi \in \operatorname{Hom}(P, \mathscr{P}(s))$, with a similar expression holding for $\gamma^{Z}\left(\sigma \cap s^{\prime}\right)$. Then we can expand

$$
\begin{aligned}
f^{X}(\tau) & =\sum_{\sigma} \mu(\sigma, \tau) \gamma^{X}(\sigma) \\
& =\sum_{\sigma} \sum_{s \subseteq \mathbf{m}} \mu(\sigma, \tau) \gamma^{Y}(\sigma \cap s) \gamma^{Z}\left(\sigma \cap s^{\prime}\right) \\
& =\sum_{\sigma} \sum_{s \subseteq \mathbf{m}} \sum_{\pi} \sum_{\rho} \mu(\sigma, \tau) \zeta(\pi, \sigma \cap s) \zeta\left(\rho, \sigma \cap s^{\prime}\right) f^{Y}(\pi) f^{Z}(\rho) \\
& =\sum_{s \subseteq \mathbf{m}} \sum_{\pi} \sum_{\rho}\left\{\sum_{\sigma} \mu(\sigma, \tau) \zeta(\pi \mid \rho, \sigma) f^{Y}(\pi) f^{Z}(\rho)\right\}
\end{aligned}
$$

since $\zeta(\pi, \sigma \cap s) \zeta\left(\rho, \sigma \cap s^{\prime}\right)=\zeta(\pi \mid \rho, \sigma)$. The last expression simplifies to

$$
\sum_{s \subseteq \mathbf{m}} \sum_{\pi} \sum_{\rho} \delta(\pi \mid \rho, \tau) f^{Y}(\pi) f^{Z}(\rho),
$$

the inner sums being over all $\pi \in \operatorname{Hom}(P, \mathscr{P}(s))$ and $\rho \in \operatorname{Hom}\left(P, \mathscr{P}\left(s^{\prime}\right)\right)$. But this is just (ii) with the interpretation given to the empty partition of $s=\varnothing$.

A more general version of this lemma is proved in exactly the same way. We state it as a corollary to the above, supposing that $U, V, \ldots$ and $W$ are similarly indexed arrays which are mutually independent, and that $X_{i}=U_{i}+V_{i}+\cdots+$ $W_{i}, i \in \mathbf{I}$.

Corollary. $f^{X}(\tau)=\sum_{\pi|\rho| \cdots \mid \sigma=\tau} f^{U}(\pi) f^{V}(\rho) \cdots f^{W}(\sigma)$, the sum being over all $\pi, \rho, \ldots, \sigma$ (including empty ones) for which $\pi|\rho| \cdots \mid \sigma=\tau$.

Our next lemma concerns arrays $X=\left(X_{i}: i \in \mathbf{I}\right)$ indexed by $\mathbf{I}=\Pi\left\{\mathbf{n}_{p}: p \in P\right\}$ for which the indices $i_{p}, p \notin a$ play no role, $a \subseteq P$ being a fixed filter of $P$. It generalises Theorem 5 of Hooke (1956a). For our later work it is convenient to suppose that $X_{i}=\varepsilon_{i}(a), i \in \mathbf{I}$, where $\varepsilon(a)=\left(\varepsilon_{i}(a): i(a) \in \mathbf{I}(a)\right)$ is a random array indexed by $\mathbf{I}(a)=\Pi\left\{\mathbf{n}_{p}: p \in a\right\}$. We then seek to express the generalised moments and cumulants of $X$ in terms of those of $\varepsilon(a)$. For a partition $\tau \in \operatorname{Hom}(P, \mathscr{P}(\mathrm{m}))$ write $\tau(a)=(\tau(p): p \in a)$ for the naturally associated element of $\operatorname{Hom}(a, \mathscr{P}(\mathbf{m}))$ obtained by restriction.

LEMMA 5.2.

(i) $\quad \gamma^{X}(\sigma)=\gamma^{\varepsilon(a)}(\sigma(a))$;

(ii) $f^{X}(\tau)= \begin{cases}f^{e(a)}(\tau(a)) & \text { if } \tau(p)=0, p \notin a, \\ 0 & \text { otherwise. }\end{cases}$

(Here 0 denotes the least element of $\mathscr{P}(\mathbf{m})$ or the number zero as appropriate.) 
Proof. To see (i) we take $h: \mathbf{m} \rightarrow \mathbf{I}$ with $\varphi^{h}=\sigma$ and observe that $h$ composed with the canonical projection from I to $\mathbf{I}(a)$ has kernel $\sigma(a)$. Thus $\gamma^{X}(\sigma)=\mathbb{E}\left\{X_{h(1)}(1) \cdots X_{h(m)}(m)\right\}=\mathbb{E}\left\{\varepsilon_{h(1)}(1) \cdots \varepsilon_{h(m)}(m)\right\}=\gamma^{\varepsilon(a)}(\sigma(a))$. We turn now to (ii), dropping the superscript on all expressions which obviously relate to $\varepsilon(a)$. Then we have

$$
\begin{aligned}
f^{X}(\tau) & =\sum_{\sigma} \mu(\sigma, \tau) \gamma^{X}(\sigma) \\
& =\sum_{\sigma}\left[\prod_{p \in P}\left\{\mu(\sigma(p), \tau(p)) \prod_{q>p} \mu(\tau(p), \sigma(q))\right\}\right] \gamma(\sigma(a)) .
\end{aligned}
$$

Now we sum over the $\sigma(p), p \in a^{\prime}=P \backslash a$, beginning with minimal elements and ensuring that, at each stage, whenever we are summing over $\sigma(p)$, the terms corresponding to $\sigma\left(p^{*}\right)$ for all $p^{*}<p$ have already been removed. Bearing in mind the fact that we have

$$
\sum_{\sigma(p)} \mu(\sigma(p), \tau(p))=\delta(0, \tau(p))
$$

we find that when $\tau(p)=0$ for all $p \in a^{\prime}$, the above expression for $f^{X}(\tau)$ reduces to $\sum_{\sigma(a)} \mu_{a}(\sigma(a), \tau(a)) \gamma(\sigma(a))$, where $\mu_{a}$ is the Möbius function of $\operatorname{Hom}(a, \mathscr{P}(\mathbf{m}))$; otherwise it reduces to zero.

The final lemma in this sequence concerns arrays $X$ for which $X_{i}=\varepsilon_{i}, i \in \mathbf{I}$, where the moments of $\varepsilon$ of order $m$ are invariant under the full symmetric group $S_{\mathbf{I}}$ over I and not just the group $G=G W(\mathbf{I})$. As before we seek expressions for the generalised moments and cumulants of $X$ in terms of those of $\varepsilon$, and this time the latter are (as in (II)) labelled by the elements of $\mathscr{P}(\mathbf{m})$.

LEMMA 5.3.

(i) $\quad \gamma^{X}(\sigma)=\gamma^{\varepsilon}(\wedge\{\sigma(p): p \in P\})$.

(ii) $f^{X}(\tau)= \begin{cases}f^{\varepsilon}(\pi) & \text { if } \tau(p)=\pi \in \mathscr{P}(\mathbf{m}) \text { for all } p \in P \\ 0 & \text { otherwise. }\end{cases}$

Proof. Since the moments of $\varepsilon$ of order $m$ are symmetric we need the kernel ker*, say, of $h: \mathbf{m} \rightarrow \mathbf{I}$ in the sense in which it was originally defined in (II), rather than as an element of $\operatorname{Hom}(P, \mathscr{P}(\mathbf{m}))$. But it is easy to see that if $\varphi^{h}=\sigma$ in the later sense, then $\operatorname{ker}^{*} h=\Lambda\{\sigma(p): p \in P\}$, the inf being taken in $\mathscr{P}(\mathbf{m})$. Thus

$$
\gamma^{X}(\boldsymbol{\sigma})=\mathbb{E}\left\{\prod_{l \in \mathbf{m}} X_{h(l)}(l)\right\}=\mathbb{E}\left\{\prod_{l \in \mathbf{m}} \varepsilon_{h(l)}(l)\right\}=\gamma^{\varepsilon}(\wedge\{\sigma(p): p \in P\})
$$

and (i) is proved. 
To obtain (ii) we note that $\zeta(\rho, \wedge\{\sigma(p): p \in P\})=\Pi_{p \in P} \zeta(\rho, \sigma(p))$ in $\mathscr{P}(\mathbf{m})$, and so, using Möbius inversion and (i) above, we have

$$
\begin{aligned}
f^{X}(\tau) & =\sum_{\sigma} \mu(\sigma, \tau) \gamma^{X}(\sigma) \\
& =\sum_{\sigma} \prod_{p \in P}\left\{\mu(\sigma(p), \tau(p)) \prod_{q>p} \mu(\tau(p), \sigma(q))\right\} \sum_{\rho} \prod_{p \in P} \zeta(\rho, \sigma(p)) f^{\varepsilon}(\rho) \\
& =\sum_{\rho} f^{\varepsilon}(\rho) \sum_{\sigma} \prod_{p \in P}\left\{\mu(\sigma(p), \tau(p)) \prod_{q>p} \mu(\tau(p), \sigma(q))\right\} \prod_{p \in P} \zeta(\rho, \sigma(p)) \\
& =\left\{\prod_{p \in P} \delta(\rho, \tau(p))\right\} f^{\varepsilon}(\rho),
\end{aligned}
$$

where the sum is simplified by summing over $\sigma(p), p \in P$, beginning with maximal elements of $P$ and, at each stage, ensuring that before summing over $\sigma(p)$, all terms involving $\sigma(q), q>p$ have been removed.

With these preliminaries completed we can state our main result concerning arrays synthesised by linear models.

Proposition 5.1. Let us suppose that $X=\left(X_{i}: i \in \mathbf{I}\right)$ is a random array given by

$$
X_{i}=\mu+\sum_{\emptyset \neq a \in F(P)} \varepsilon_{i(a)}, \quad i \in \mathbf{I},
$$

where for all $\varnothing \neq a \in F(P)$,

(i) $\varepsilon(a)=\left(\varepsilon_{i(a)}: i(a) \in \mathbf{I}(a)\right)$ is a random array indexed by $\mathbf{I}(a)=\Pi\left\{\mathbf{n}_{p}: p \in\right.$ a);

(ii) the moments of order $m$ of $\varepsilon(a)$ are symmetric; and

(iii) the arrays $\{\varepsilon(a)\}$ are mutually independent.

Then $X$ has $G W$-invariant moments of order $m$ and for every $\tau \in \operatorname{Hom}(P, \mathscr{P}(\mathbf{m}))$ we have

$$
f^{X}(\tau)=\sum f^{\varepsilon\left(a_{1}\right)}\left(\pi_{1}\right) f^{\varepsilon\left(a_{2}\right)}\left(\pi_{2}\right) \cdots
$$

where the sum is over all $\pi_{1}, \pi_{2} \cdots \in \mathscr{P}(\mathbf{m})$ for which the elements $\sigma_{1}, \sigma_{2}, \ldots$ of $\operatorname{Hom}(P, \mathscr{P}(\mathbf{m}))$ given by

$$
\sigma_{i}(p)= \begin{cases}\pi_{i} & \text { if } p \in a_{i}, \\ 0 & \text { otherwise }\end{cases}
$$

$i=1,2, \ldots$, satisfy $\sigma_{1} \mid \sigma_{2} \cdots=\tau$.

COROLlary. Suppose that $m=2$. Then if for every $\sigma \in \operatorname{Hom}(P, \mathscr{P}(2))$ we write $a(\sigma)=\{p \in P: \sigma(p)=12\}$, we have

$$
f^{X}(\sigma)=f^{\varepsilon(a(\sigma))}(12)
$$


Proof. This is an immediate consequence of the proposition, for every $\sigma \in$ $\operatorname{Hom}(P, \mathscr{P}(2))$ is uniquely determined by $a(\sigma)$.

The corollary asserts that when $m=2$, each of the generalised cumulants associated with $X$ defined by such a linear model is a generalised cumulant of a component array. If the elements of the component arrays are themselves independent, then this is just a (co)variance, and so our generalised cumulants reduce to components of (co)variance with the usual linear models. Further aspects of this connexion will be explored in the next section.

EXAMPLE 5.1. Let $P=! \frac{1}{2}$. The linear model naturally associated with this nesting poset is

$$
X_{i j}=\mu+\varepsilon_{i}(1)+\varepsilon_{i j}(1,2)
$$

where $\mu$ is constant, the sets $\left\{\varepsilon_{i}(1): i \in \mathbf{n}_{1}\right\}$ and $\left\{\varepsilon_{i j}(1,2):(i, j) \in \mathbf{n}_{1} \times \mathbf{n}_{2}\right\}$ of effects are independent, and exchangeable (if not mutually independent) within sets. The generalised cumulant $f^{X}(14|23,14| 2 \mid 3)$ appears in the expression for the variance of $k(12,1 \mid 2)$ (the between class component of variance in the anova table) and so it is of interest to compute this cumulant in terms of those of $\varepsilon(1)$ and $\varepsilon(1,2)$. Using Proposition 5.1 we expand as follows:

$$
\begin{aligned}
f^{X}(14|23,14| 2 \mid 3)= & f^{\varepsilon(1)}(14|23,14| 2 \mid 3)+f^{\varepsilon(1)}(14,14) f^{\varepsilon(1,2)}(23,2 \mid 3) \\
& +f^{\varepsilon(1,2)}(14,14) f^{\varepsilon(1)}(23,2 \mid 3)+f^{\varepsilon(1,2)}(14|23,14| 2 \mid 3) \\
= & f^{\varepsilon(1,2)}(14) f^{\varepsilon(1)}(23)
\end{aligned}
$$

for $f^{\varepsilon(1)}(14|23,14| 2 \mid 3)=0$ since $14|2| 3 \neq 1|2| 3 \mid 4$ (the zero of $\mathscr{P}(4)$ ), $f^{\varepsilon(1)}(14,14)=0$ for a similar reason, whilst $f^{e(1,2)}(14|23,14| 2 \mid 3)=0$ since $14 \mid 23$ $\neq 14|2| 3$.

\section{Components of variances}

We have asserted on a number of occasions that our generalised symmetric functions $k(\sigma), \sigma \in \operatorname{Hom}(P, \mathscr{P}(2))$ coincide with familiar sample components of variance from the analysis of variance of the multi-indexed array $\left(X_{i}: i \in \mathbf{I}\right)$. This result, which was conjectured by Zyskind (1958) and first proved by Dayhoff $(1964 \mathrm{a}, \mathrm{b})$ is easy to prove here using the machinery now available to us: all we need to do is rearrange our notation so that the tensors $A_{\sigma}, R_{\sigma}$ and $F_{\sigma}$ are in fact $n \times n$ matrices, where $n=\Pi\left\{n_{p}: p \in P\right\}$, and use some general results.

If, instead of defining $\bar{h}=\delta^{h(1)} \otimes \delta^{h(2)}$ as we did in Section 2, we write $\bar{h}=\delta^{h(1)}\left(\delta^{h(2)}\right)^{\prime}$, then $\bar{h}$, and hence $A_{\sigma}, R_{\sigma}$ and $F_{\sigma}$, would all be $n \times n$ matrices over I, where $n=|\mathbf{I}|=\Pi\left\{n_{p}: p \in P\right\}$. Let us suppose this to be the case for the 
remainder of this section. Then the family $\left\{A_{\sigma}: \sigma \in \operatorname{Hom}(P, \mathscr{P}(2))\right\}$ of matrices defines an association scheme over $\mathbf{I}$. The simplest way to see this is to observe that the $\left\{A_{\sigma}\right\}$ are simply the characteristic (indicator) functions of the orbits of the action of $G W(\mathbf{I})$ on $\mathbf{I} \times \mathbf{I}$, and since these orbits are all self-paired, the assertion follows (cf. Higman $(1975,1976)$ ). An alternative proof can be based upon the results in Speed and Bailey (1982), the contents of which will be helpful in what follows.

Once we have established that the $\left\{A_{\sigma}\right\}$ form an association scheme, we know that they can be simultaneously diagonalised, and that the orthogonal projections onto their common eigenspaces can all be written as linear combinations of the $\left\{A_{\sigma}\right\}$; see for example MacWilliams and Sloane (1978). In this case the orthogonal projections are also indexed by $\operatorname{Hom}(P, \mathscr{P}(2))$ (see Speed and Bailey (1982)), and we denote them by $\left\{S_{\sigma}: \sigma \in \operatorname{Hom}(P, \mathscr{P}(2))\right\}$. Thus there exist matrices $P=\left(p_{\sigma \rho}\right)$ and $Q=\left(q_{\rho \sigma}\right)$ such that

$$
\begin{gathered}
S_{\sigma}=\frac{1}{n} \sum_{\rho} q_{\rho \sigma} A_{\rho}, \\
A_{\rho}=\sum_{\rho} p_{\sigma \rho} S_{\sigma} .
\end{gathered}
$$

Now $d_{\sigma}=\operatorname{rank}\left(S_{\sigma}\right)$ is called the degrees of freedom of the stratum labelled by $\sigma$, and an important related coefficient is $k_{\rho}=n^{-1}(n)_{\rho}$, for we know the identity

$$
d_{\sigma}^{-1} q_{\rho \sigma}=k_{\rho}^{-1} p_{\sigma \rho},
$$

(see McWilliams and Sloane (1978, eqn. (18) of Theorem 3, Chapter 21, Section 2)). One final fact which we need is a result from Speed and Bailey (1982), namely,

$$
p_{\sigma \rho}=\frac{1}{n} \sum_{\pi} \mu(\rho, \pi) \zeta(\sigma, \pi) n^{\pi}
$$

The assertion that we wish to prove really has two forms: one involving generalised symmetric functions and one involving generalised cumulants. Because they were always operating within the framework where all distributions were permutation distributions associated with finite populations, essentially a special case of our srswor set-up, these two forms coincided for Zyskind and his successors. A form which includes both is the following.

PROPOSITION 6.1. In the notation just introduced,

$$
F_{\tau}=\frac{n}{n^{\tau}} \sum_{\sigma} \mu(\sigma, \tau) d_{\sigma}^{-1} S_{\sigma}
$$


Proof. We substitute (6.1) into the right hand side of the above, and use (6.3), (6.4) and Möbius inversion to get our result:

$$
\begin{aligned}
& \frac{n}{n^{\tau}} \sum_{\sigma} \mu(\sigma, \tau) d_{\sigma}^{-1} S_{\sigma}=\frac{n}{n^{\tau}} \sum_{\sigma} \mu(\sigma, \tau) d_{\sigma}^{-1} \frac{1}{n} \sum_{\rho} q_{\rho \sigma} A_{\rho} \\
& =\frac{1}{n^{\tau}} \sum_{\sigma} \sum_{\rho} \mu(\sigma, \tau) k_{\rho}^{-1} p_{\sigma \rho} A_{\rho} \\
& =\frac{1}{n n^{\tau}} \sum_{\sigma} \sum_{\rho} \sum_{\pi} \mu(\sigma, \tau) \mu(\rho, \pi) \zeta(\sigma, \pi) n^{\pi} k_{\rho}^{-1} A_{\rho} \\
& =\sum_{\rho} \mu(\rho, \tau)\left(n k_{\rho}\right)^{-1} A_{\rho} \\
& \text { (by Möbius inversion) } \\
& =F_{\tau} \\
& \left(\text { since } n k_{\rho}=(n)_{\rho}\right. \text { ) }
\end{aligned}
$$

COROLLARY. (i) If $\left[S_{\sigma} X(1) \mid S_{\sigma} X(2)\right]$ is the sum of products in the line (stratum) labelled by $\sigma \in \operatorname{Hom}(P, \mathscr{P}(2))$ of the anova table, then

$$
k_{\tau}=\frac{n}{n^{\tau}} \sum_{\sigma} \mu(\sigma, \tau) d_{\sigma}^{-1}\left[S_{\sigma} X(1) \mid S_{\sigma} X(2)\right] .
$$

(ii) If $\xi_{\sigma}=\mathbb{E}\left\{d_{\sigma}^{-1}\left[S_{\sigma} X(1) \mid S_{\sigma} X(2)\right]\right\}$ is the expected mean sum of products in line $\sigma$, then

$$
f_{\tau}=\frac{n}{n^{\tau}} \sum_{\sigma} \mu(\sigma, \tau) \xi_{\sigma}
$$

Proof. The result (i) is obtained directly from the proposition by introducing the elements $X=(X(1), X(2))$ of the random array as indeterminates, and (ii) follows by taking expectations. Either can be construed as stating Zyskind's conjecture when $X(1)=X(2)$, for under a randomisation model they coincide.

We close this section with some remarks concerning the estimation of the variance components $f(\sigma)$ of an array $X=\left(X_{i}: i \in \mathbf{I}\right)$, recalling that elements of $\operatorname{Hom}(P, \mathscr{P}(\dot{2}))$ are more readily labelled by the filters of $P$. Under an assumption of $G W$-invariance of the moments of order 4, we can conclude from Proposition 4.2 that the symmetric functions $k(\sigma)$ provide minimum variance estimators of $f(\sigma)$. If we specialise this result to the linear model framework discussed in the previous section and further suppose that the effects $\left(\varepsilon_{i(a)}: i(a) \in \mathbf{I}(a)\right)$ are 
mutually independent, $\varnothing \neq a \in F(P)$, this assertion reduces to a result of Graybill and Hultquist (1959, Theorem 7) concerning MIVQUE of variance components.

\section{Closing remarks}

Two topics not covered here will be dealt with in later papers in this series. The first concerns the fact that all the generalised $k$-statistics, and the other generalised symmetric functions, are multi-indexed reversed martingales with respect to certain naturally defined arrays of $\sigma$-fields. These reversed martingales turn out to satisfy the further conditions necessary to ensure their a.s. covergence and a particular consequence of this is a neat asymptotic theory for variance component estimates. The second topic concerns the form and estimates of the variances and covariances of estimated variance components. Because of its special interest and algebraic complexity, this topic warrants a paper in its own right.

Further results which may be published later concern the same issues with differently structured index sets such as those which arise in quantitative genetics; see for example Nelder (1960).

In closing it is a pleasure to thank Professor Oscar Kempthorne most warmly for providing copies of the relevant Iowa theses done under his direction. Without having had access to this largely unpublished body of fine material, I would not have been able to do this work. Thanks are also due to the referees for their helpful comments and for providing further relevant references.

\section{References}

R. A. Bailey, C. E. Praeger, C. A. Rowley and T. P. Speed (1983), 'Generalised wreath products of permutation groups', Proc. London Math. Soc. (3) 47, 69-82.

Edward John Carney (1967), Computation of variances and covariances of variance component estimates, $\mathrm{Ph} \mathrm{D}$ Thesis, Iowa State University.

E. J. Carney (1968), 'Relationship of generalised polykays to unrestricted sums for balanced complete finite populations', Ann. Math. Statist. 39, 643-656.

E. J. Carney (1970), 'Multiplication of polykays using ordered partitions', Ann. Math. Statist. 41, $1749-1752$.

Jerome Cornfield and John W. Tukey (1956), 'Average values of mean squares in factorials', Ann. Math. Statist. 27, 907-949.

Eugene Dayhoff (1964a), Generalised polykays and application to obtaining variances and covariances of components of variation, $\mathrm{Ph} \mathrm{D}$ Thesis, Iowa State University.

Eugene Dayhoff (1964b), 'On the equivalence of polykays of the second degree and $\Sigma$ 's', Ann. Math. Statist. 35, 1663-1672.

Eugene Dayhoff (1966), 'Generalised polykays, an extension of simple polykays and bipolykays', Ann. Math. Statist. 37, 226-241. 
Peter Doubilet (1972), 'On the foundations of combinatorial theory VII: Symmetric functions through the theory of distribution and occupancy', Stud. Appl. Math. 11, 377-396.

H. Fairfield Smith (1955), 'Variance components, finite populations and experimental inference', North Carolina Institute of Statistics Mimeo Series No. 135.

Franklin A. Graybill and Robert A. Hultquist (1960), 'Theorems concerning Eisenhart's Model II', Ann. Math. Statist. 31, 261-269.

D. G. Higman (1975), 'Coherent configurations I', Geom. Dedicata 4, 1-32.

D. G. Higman (1976), 'Coherent configurations II', Geom. Dedicata 5, 413-424.

Robert Hooke (1956a), 'Symmetric functions of a two-way array', Ann. Math. Statist. 27, 55-79.

Robert Hooke (1956b), 'Some applications of bipolykays to the estimation of variance components and their moments', Ann. Math. Statist. 27, 80-98.

Oscar Kempthorne (1952), The design the analysis of experiments (John Wiley \& Sons, Inc., New York).

John Kinney (1971), Multivariate and generalised polykays in statistical structures, Ph D Thesis, Iowa State University.

John Kinney (1976), 'The $k$-statistics, polykays and randomised sums', Sankhya $\operatorname{Ser}$. $A$ 38, 271-286.

F. J. MacWilliams and N. J. A. Sloane (1978), The theory of error correcting codes (North-Holland Publishing Company, Amsterdam, New York).

J. A. Nelder (1960), 'The estimation of variance components in certain types of experiments in quantitative genetics', Biometrical Genetics, edited by O. Kempthorne (London, Pergamon Press).

C. E. Praeger, C. A. Rowley and T. P. Speed (1985), 'A note on generalised wreath product groups', $J$. Austral Math. Soc. (Ser. A) 39, 415-420.

T. P. Speed and R. A. Bailey (1982), 'On a class of association schemes derived from lattices of equivalence relations', Algebraic Structures and their Applications, edited by Philip Schultz, Cheryl E Praeger and Robert P. Sullivan (Marcel Dekker, New York).

T. P. Speed (1983), 'Cumulants and partition lattices', A ustral. J. Statist. 25, 378-388.

T. P. Speed (1984), 'On the Möbius function of $\operatorname{Hom}(P, Q)$ ', Bull. Austral. Math. Soc. 29, 39-46.

T. P. Speed (1985), 'Cumulants and partition lattices II. Generalised $k$-statistics', J. Austral. Math. Soc. (Ser. A), 40, 34-53.

T. P. Speed (1986), 'Anova models with random effects: an approach via symmetry", to appear in the E. J. Hannan Festschrift Volume, J. Appl. Probab.

Thomas Neil Throckmorton (1961), Structures of classification data, Ph D Thesis, Iowa State University.

R. F. White (1963), Randomisation analysis of the general experiment, $\mathrm{Ph} \mathrm{D}$ Thesis, Iowa State University.

R. F. White (1975), 'Randomisation and the analysis of variance', Biometrics 31, 552-572.

M. B. Wilk (1955), 'The randomisation analysis of a generalised randomised block design', Biometrika 42, 70-79.

M. B. Wilk and O. Kempthorne (1956), 'Some aspects of the analysis of factorial experiments in a completely randomised design', Ann. Math. Statist. 27, 950-985.

M. B. Wilk and Oscar Kempthorne (1957), 'Non-additivities in a Latin square design', J. Amer. Statist. Assoc. 52, 218-236.

George Zyskind (1958), Error structure in experimental design, $\mathrm{Ph} D$ Thesis, Iowa State University.

George Zyskind (1962), 'On structure, relation, $\Sigma$, and expectation of mean squares', Sankhyā Ser. A 24, 115-148.

\section{CSIRO Division of Mathematics and Statistics}

Box 1965 GPO

Canberra, A.C.T. 2601

Australia 